mètre cube. A la fin de l'expérience, la teneur bactérienne moyenne, déterminée par culture sur plaques, du lait, lors de la livraison au dépôt, était inférieure à 6.800 unités par centimètre cube. Pour neuf échantillons sur douze, il ne fut pas possible de déterminer la présence du $B$. Coli dans $1 \mathrm{~cm}^{2}$, et les teneurs moyennes à ce sujet furent inférieures à celles de la fourniture à la Société de lait "Grade A. Tuberculin Tested" (catégorie A, de vaches tuberculinées) du même mois. Tenant compte des faits déjà exposés, les résultats les plus significatifs furent, peut-être, ceux obtenus après la pasteurisation. Avant l'expérience, la teneur bactérienne moyenne, après la pasteurisation de laboratoire des fournitures des douze fermiers, était de 27.000 unités par centimètre cube. A la fin de la période d'expérience, on obtint des plaques nettes après la pasteurisation pour les fournitures de six fermiers. La teneur bactérienne moyenne pour les douze participants ne fut que de 23 organismes par centimètre eube, tandis que la plus élevée, qui ne fut constatée que dans un seul cas, fut de 100 unités par centimètre cube.

(A suivre.)

\title{
EMPLOI DE LA TEINTURE DE GAIAC DANS LE CONTROLE DE LA PASTEURISATION
}

\author{
par B. LEIBOVITCH
}

Ingénieur Chimiste (A. I. G.). Diplômé de Microbiologie. Chef du Laboratoire de la Laiterie Saint-Hubert, Naney.

En 1820, Planche observe pour la première fois la coloration bleue du lait cru en y ajoutant quelques gouttes de teinture de gaïac (BARTHEL), mais e'est seulement en 1881 que ARnold se sert de ce réactif pour différencier un lait cru d'un lait pasteurisé. Cette réaction d'Arnold, comme beaucoup d'autres (Dupouy, Storch, Rothenfusser), est basée sur la destruction par la chaleur de la peroxydase se trouvant dans le lait cru. L'utilisation de ces réactions dans le contrôle de la pasteurisation présente un certain nombre d'avantages : elles sont généralement d'un emploi facile et nous indiquent en quelques minutes si nous nous trouvons en présence d'un lait eru ou d'un lait pasteurisé. Cette rapidité d'appréciation permet d'écarter de la consommation tout lait ne correspondant pas aux conditions prescrites.

Malheureusement, ces contrôles biochimiques et physicochimiques ne sont pas suffisamment sensibles : une addition de 3 à $5 \%$ de lait cru au lait pasteurisé, ne peut pas être décelée avec certitude. La réaction de Schern-Gorli, étudiée et modifiée par TAPERnoux [1], Pien et Baisse [2] et Philippe [3], reste même insensible à toute addition de lait cru écrémé au lait pasteurisé ; cette réaction 
étant basée sur le changement des propriétés physico-chimiques des globules gras du lait sous l'action de la chaleur. Or, de telles additions compromettent fatalement la qualité du lait pasteurisé. Un autre point faible du contrôle biochimique : il ne décèle pas les contaminations microbiennes provenant d'un manque d'hygiène dans le travail. La généralisation de méthodes biochimiques de contrôle à l'exclusion des méthodes bactériologiques pourrait même présenter un danger pour l'avenir de l'industrie laitière. M. Guittonnead [4] a cru utile d'attirer l'attention sur cette question en demandant "que dans tous les cas où s'imposent des considérations d'ordre bactériologiques, on n'hésitât pas à faire appel aux vraies techniques bactériologiques qui conduisent à des résultats infiniment plus clairs et infiniment plus sûrs que tous ces contrôles indirects, dont on a tant abusé, et qui, trop souvent, n'ont fait que paralyser les progrès techniques réels en créant des atmosphères de fausse sécurité ").

Il est indiscutable que seules les techniques bactériologiques conviennent pour étudier et contrôler le fonctionnement de l'ensemble d'une installation de pasteurisation. Encore faut-il tenir compte des faits suivants : le contrôle bactériologique est trop. long ; il se limite généralement à la recherche des microbes saprophytes et du colibacille; il ne recherche pas les vrais germes pathogènes et il ne peut donner aucune indication sur le traitement thermique subi par le lait. C'est pourquoi, dans le contrôle de tous les jours, une méthode permettant de s'assurer rapidement et avec certitude que le lait a réellement subi un traitement thermique suffisant pour la destruction de tous les germes pathogènes, serait d'une grande utilité. Il faut done faire appel à ces méthodes biochimiques et surtout augmenter leur sensibilité.

L'exemple d'un contrôle biochimique parfait nous est donné par Kay et Graham [5]. Ces Auteurs ont réussi, par la recherche de la phosphatase, à déceler une addition de $0,25 \%$ de lait cru au lait pasteurisé à $63^{\circ}$ pendant $30^{\prime}$. Nous avons vérifié cette méthode ; elle donne de bons résultats pour la pasteurisation basse, mais pour la pasteurisation haute sa sensibilité est nettement atténuée.

Il nous semble que la grande sensibilité de la réaction de KAY et GrahaM dans la pasteurisation basse, s'explique par le fait que la température et la durée de chauffage de la pasteurisation basse correspondent précisément aux minima de conditions nécessaires pour la destruction de la phosphatase; la réaction devient moins sensible quand on dépasse ces conditions.

Nous avons pensé qu'il existait des conditions analogues pour la destruction de la peroxydase dans la pasteurisation haute et augmenter ainsi la sensiblité des méthodes basées sur sa recherche. 
La présente étude a pour objet de déterminer :

a) Les conditions précises de traitement thermique du lait pour lesquelles les réactions basées sur la recherche de la peroxydase deviennent sensibles à une addition de $1 \%$ de lait cru.

b) Et en particulier les conditions d'utilisation de la teinture de gaïac dans le contrôle du lait ainsi traité.

Cette étude comprend trois parties :

1. Détermination du traitement thermique subi par le lait dans une pasteurisation haute.

2. Analyse bactériologique de laits pasteurisés à des températures de $75^{\circ}$ à $81^{\circ}$.

3. Etude de la teinture de gaïac ; sa préparation et son emploi dans le contrôle de la pasteurisation.

\section{DETERMINATION DU TRAITEMENT THERMIQUE} SUBI PAR LE LAIT DANS LA PASTEURISATION HAUTE.

On ne trouve que rarement des précisions sur le traitement thermique du lait dans la pasteurisation haute. On admet généralement que le lait est porté à haute température $\left(80^{\circ}-90^{\circ}\right)$ pendant quelques secondes. En réalité, il s'agit souvent de nombreuses secondes. OrLA-Jensen et Winter [6] constatent que ce point est "resté constamment un peu obscur " et ils rappellent, à propos, "que dans les anciens appareils de pasteurisation, la durée de chauffage était souvent de quelques minutes ". On conçoit facilement la différence entre un chauffage de quelques secondes et un chauffage de quelques minutes à la même température. On pourrait faire une remarque analogue sur la température de pasteurisation : cette température n'est nullement constante pendant la durée du chauffage, elle varie avec le temps. Le seul moyen correct de préciser le traitement thermique d'une pasteurisation haute c'est de tracer la courbe des températures en fonction du temps. Nous avons déterminé cette courbe pour le pasteurisateur qui a servi à nos expériences, à la Laiterie SaintHubert, de Nancy.

C'est un appareil à plaques (A. P. V.) comportant un chambreur et une grosse plaque servant également

GRAPHIQUE I

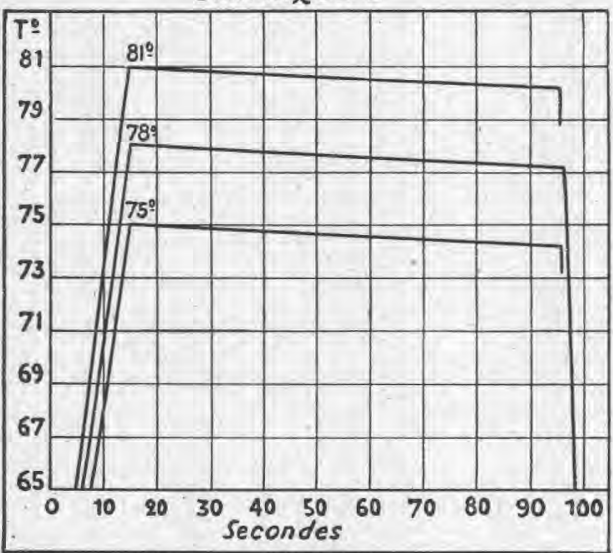


au chambrage du lait. Les températures ont été obtenues avec des thermomètres contrôlés, installés aux principaux points du trajet suivi par le lait. Nous avons calculé les temps en tenant compte du débit et du volume de différentes parties de l'appareil se trouvant entre deux thermomètres. En faisant varier la température de pasteurisation, on obtient une famille de courbes qui sont, dans leurs parties les plus importantes, pratiquement parallèles (voir graphique 1). Quand nous parlerons, au cours de cet article, d'un chauffage à $78^{\circ}$, le traitement thermique sera défini par la courbe dont le maximum passe par $78^{\circ}$. Ces courbes sont caractéristiques pour chaque type de pasteurisateur.

\section{ANALYSE BACTÉRIOLOGIQUE DE LAITS PASTEURISÉS A DES TEMPÉRATURES DE $75^{\circ}$ à $81^{\circ}$.}

Nous avons procédé à cinq essais de pasteurisation à des températures variant de $75^{\circ}$ à $81^{\circ}$. Pour des raisons d'exploitation, nous n'avons pas eu la possibilité d'utiliser un seul mélange de lait pour les différentes pasteurisations d'un essai. Seuls, les laits pasteurisés à $77^{\circ}, 78^{\circ}$ et $79^{\circ}$ proviennent d'un même mélange de lait cru. Ce lait cru fut analysé et les résultats sont donnés dans le tableau 1.

Le pasteurisateur a été stérilisé avant le travail, en faisant circuler en circuit fermé, pendant 30 minutes, de l'eau formolée et chauffée à $75^{\circ}$.

Pour ne pas contaminer l'appareil, nous commencions par la pasteurisation à $81^{\circ}$ pour descendre graduellement à $75^{\circ}$.

TABLEAU 1 .

\begin{tabular}{|c|c|c|c|c|c|c|c|c|}
\hline & \multicolumn{8}{|c|}{ Température de pasteurisation } \\
\hline & $81^{\circ} \mathrm{C}$, & $80^{\circ}$ & $79^{\circ}$ & $78^{\circ}$ & $77^{\circ}$ & $76^{\circ}$ & $75^{\circ}$ & Lait cru \\
\hline 1 & $\mathrm{E}(1)$ & 1.540 & 4.200 & 6.200 & 63.600 & 71.600 & 117.500 & 16.400 .000 \\
\hline 2 & E & $\mathrm{E}$ & 3.700 & 6.100 & 46.000 & 111.000 & 114.800 & $13,600,000$ \\
\hline 3 & 300 & 800 & 4.100 & 5.100 & 36.500 & 69.200 & 176.000 & 9.200 .000 \\
\hline 4 & $\mathrm{E}$ & 1.440 & 13.100 & 23.200 & 87.000 & 98.000 & 192.000 & 12.300 .000 \\
\hline \multirow[t]{3}{*}{$\tilde{\jmath}$} & 200 & 500 & 800 & 4.200 & 54.400 & 83.200 & 121.000 & 10.400 .000 \\
\hline & \multicolumn{8}{|c|}{ Moyennes des résultats de 5 expériences } \\
\hline & 250 & 1.070 & 5.180 & 8.960 & 57.500 & 86.600 & $144.260 \mid$ & 12.380 .000 \\
\hline
\end{tabular}

(1) Toutes les boîtes de Petri ensemencées ont été envahies par des colonies de bacilles thermorésistants empêchant le développement des autres colonies. On peut limiter de tels envahissements en réduisant la température de l'étuve à $25^{\circ}-30^{\circ}$. Mais nous n'avons pas voulu nous écarter de nos méthodes de travail habituelles, les résul. tats de la présente étude devant nous servir d'éléments de comparaison dans notre travail futur de contrôle. 
Tous les laits ont été analysés ; le nombre de microbes au centimètre cube a été déterminé en ensemençant des boîtes de Petri et en comptant les colonies après 48 heures d'étuve à $37^{\circ}$. Les ensemencements ont été effectués avec le milieu de culture d'Orla-Jensen sur gélose. Nous avons recherché également le colibacille dans $0,01,0,1,1,2$ et $5 \mathrm{~cm}^{3}$ de lait. Tous les milieux de culture qui ont servi à ces analyses furent préparés en une seule fois. Dans tous les laits crus, le colibacille a été présent dans $0 \mathrm{~cm}^{3} 01$. Dans tous les laits pasteurisés le colibacille était absent dans $5 \mathrm{~cm}^{3}$. Les nombres de microbes trouvés au centimètre cube sont donnés dans le tableau 1 .

En traçant la courbe des moyennes des résultats de cinq expériences, on se rend compte de l'allure de ce phénomène (voir graphique 2). 11 est bien entendu que cette courbe ne peut exprimer graphiquement toute la vérité du phénomène, mais en donne seulement une idée. Ayant fait cette réserve, on constate que la température de $78^{\circ}$ est critique pour la presque totalité des germes se trouvant dans le lait eru. Nous verrons que cette température est également critique pour la peroxydase du lait. C'est cette température qui a été choisie pour notre pasteurisation. Les températures de $76^{\circ}$ et $77^{\circ}$ correspondraient mieux à ce que GoRINI $[7,8]$ appelle avec raison la " pas-

GRAPHIQUE II

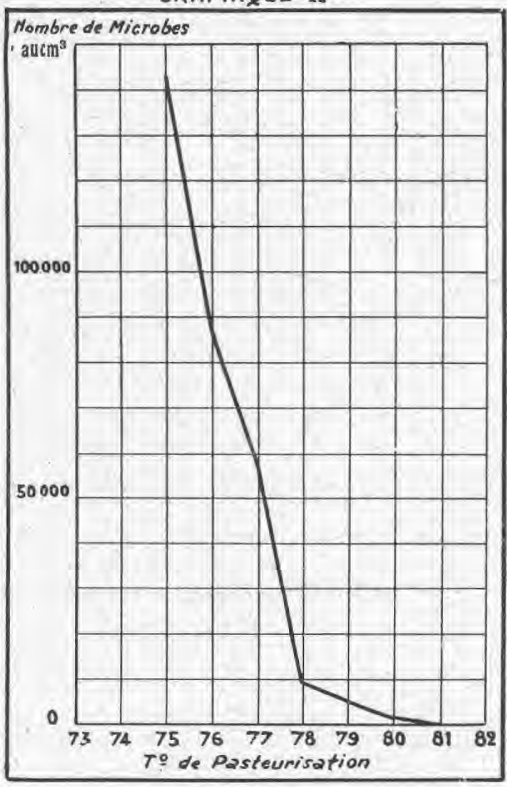
teurisation rationnelle" du lait. Le rapport entre les germes thermorésistants acidifiants et les thermorésistants sporogènes serait dans ee cas plus rationnel. Mais l'accroissement du nombre de microbes restant dans le lait est trop important pour ces températures.

\section{ETUDE DE LA TEINTURE DE GAIAG : SA PREPARATION ET SON EMPLOI DANS LE GONTROLE DE LA PASTEU- RISATION.}

La teinture de gaïac du commerce est généralement inactive et ne donne aucune réaction avec le lait cru. Nous nous sommes servis pour nos premières études d'une teinture de gaïac astive, provenant d'un laboratoire spécialisé en Allemagne. Une teinture préparée, 
d'après BARTHEL [9], avec du bois de gaïac râpé et de l'acétone était inactive. M. le Professeur A. TAPERnoux nous a conseillé d'essayer la résine de gaïac. Calmette, NÈGRe et Boquet [10] indiquent également une formule de préparation à base de résine de gaïac. La résine de gaïas ne nous a pas donné de meilleurs résultats. C'est en multipliant les essais que nous avons ajouté un peu de gaïacol cristallisé dans la teinture préparée avec de la résine de gaïac et alors elle est devenue immédiatement active. La teinture préparée avec du bois de gaïac devient également active, par l'addition d'un peu de gaïacol.

La teinture ainsi préparée est restée pour nous [11] le réactif d'Arnold. Mais nous trouvons chez VAN DEN BURG [12] qu'un réactif portant le nom de Schern et Sehellhase est "constitué par de la résine de gaïac et du gaïacol ». MASEK [13] parle, lui aussi, de la réaction de Schern et Schellhase et on peut déduire de la bibliographie indiquée que ces Auteurs ont publié leurs travaux en 1911. Nous n'avons pas pu, malheureusement, nous procurer les travaux de Schern et Schellhase.

Par la suite, nous avons réussi à préparer une teinture de gaïac active, uniquement avec de la résine de gaĩac ; on a plus de chance de réussir en choisissant parmi les gros moreeaux de résine de gaïac. Cette teinture est peu sensible.

\section{Préparation d'une teinture de gaïac active et son mode d'emploi.}

On dissout un gramme de résine de gaïac dans $100 \mathrm{~cm}^{3}$ d'acétone, on filtre et on ajoute $0 \mathrm{gr}$. 5,1 ori 3 grammes de gaïacol cristallisé, suivant que l'on désire une teinture peu ou fortement sensible.

Pour pratiquer l'essai, on verse dans un tube de $15 \mathrm{~mm}$. de diamètre, $10 \mathrm{~cm}^{3}$ de lait et une goutte $\left(0 \mathrm{~cm}^{3} 05\right)$ d'eau oxygénée à 3 volumes, on mélange et on ajoute $1 \mathrm{~cm}^{3}$ de teinture de gaïac. On secoue le tube pour obtenir un mélange de la teinture avec la partie supérienre du lait. La réaction est positive quand la partie supérieure du lait (environ $1 \mathrm{~cm}$. en profondeur) s'est colorée en bleu; on note le temps. Lorsque la réaction ne se produit pas en 30 minutes, elle est considérée comme négative.

Pour obtenir une réaction positive, il faut, semble-t-il, que les 4 facteurs suivants soient réunis :
1. Un peroxyde $\left(\mathrm{H}^{2} \mathrm{O}^{2}\right)$.
2. La résine de gaïac.
3. Le gaïacol et
4. La peroxydase.

Dans certains cas la résine de gaïac seule donne des teintures 
actives parce qu'elle contient un peu de gaïacol ; ces teintures sont peu sensibles parce qu'elles contiennent trop peu de gaïacol.

Certaines teintures de gaïac sont actives sans addition d'eau oxygénée, elles contiennent, d'après Inıкноv [14], des peroxydes formés sous l'action de la lumière.

$$
\text { *** }
$$

Pour déterminer la formule de préparation d'une teinture de gaïac active et adaptée au contrôle des laits pasteurisés à $78^{\circ}$, nous avons été amené à étudier l'influence de chacun des facteurs indiqués précédemment.

\section{Rôle de l'eau oxygénée.}

Il n'est pas indifférent d'employer une faible ou une forte quantité d'eau oxygénée : des essais résumés dans le tableau 2 il résulte que la meilleure dose c'est $0 \mathrm{~cm}^{3} 025$ d'eau oxygénée à 3 volumes. Mais les solutions d'eau oxygénée étant peu stables, leur force diminuant avec le temps, nous avons préféré adopter la dose de $0 \mathrm{~cm}^{3} 05$ (une goutte). Ceci nous permet de renouveler l'eau oxygénée tous les 15 jours seulement.

Tablead 2.

\begin{tabular}{|c|c|c|c|}
\hline Nature du lait & $\begin{array}{l}\text { Quantité de } \mathrm{H}^{2} \mathrm{O}^{2} \\
\text { à } 3 \text { volumes ajouté } \\
\text { (centimètres cubes) }\end{array}$ & Durée de la réaction & Observations \\
\hline $\begin{array}{l}\text { Lait pasteurisé à } 78^{\circ} \\
\text { contenant } 1 \% \text { de lait } \\
\text { eru. }\end{array}$ & $\begin{array}{l}0,25 \\
0,10 \\
0,05 \\
0,025 \\
0,005 \\
0,0025\end{array}$ & $\begin{array}{l}\text { + après } 16^{\prime} \\
+ \text { après } 4^{\prime} 45^{\prime \prime} \\
\text { + après } 3^{\prime} 15^{\prime \prime} \\
\text { + après } 2^{\prime} 30^{\prime \prime} \\
\text { + après } 3^{\prime} 15^{\prime \prime} \\
\text { + après } 12^{\prime}\end{array}$ & $\begin{array}{l}\text { bleu pâle } \\
\text { bleu foncé } \\
\text { bleu foncé } \\
\text { bleu foncé } \\
\text { bleu foncé } \\
\text { bleu pâle }\end{array}$ \\
\hline
\end{tabular}

\section{Rôle de la résine de gaïac.}

Nous avons préparé des teintures à concentrations en résine de gaïac variables. Les essais sont résumés dans le tableau 3.

Une forte dose de résine de gaïac a une action inhibante sur la réaction, tandis que les faibles doses provoquent des réactions plus rapides, mais aussi peu stables, la coloration bleue disparaissant rapidement. Nous avons adopté la dose de $1 \%$ de résine de gaĩac, produisant des colorations bleues foncées, rapides et stables.

Les teintures contenant moins de $1 \%$ de résine de gaïac donnent avec le lait cru des colorations bleues se transformant rapidement 
Gaïacol $3 \%$, résine de gaïac $6 \%$ Gaïacol $3 \%$, résine de gaïac $3 \%$ Gaïacol $3 \%$, résine de gaïac $1 \%$ Gaïacol $3 \%$, résine de gaïac $0,5 \%$ Gaïacol $3 \%$, résine de gaïac $0,25 \%$ Gaïacol $3 \%$, résine de gaïac $0,12 \%$ Gaïacol $3 \%$, résine de gaïac $0,06 \%$ Gaïacol $3 \%$, résine de gaïac $0,03 \%$ Gaïacol $3 \%$, résine de gaïac $0,008 \%$ Gaïacol $3 \%$, résine de gaïac $0,004 \%$ Gaïacol $3 \%$, résine de gaïac $0,0 \%$.

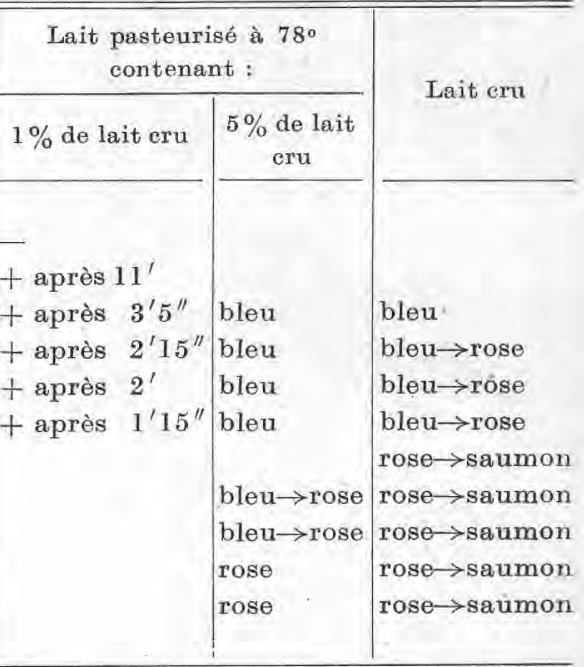

en rose. Les teintures contenant moins de $0,12 \%$ de résine de gaïac donnent avec le lait cru des colorations rose-saumon : nous retrouvons ici la classique réaction de Dupouy.

\section{Rôle du gaïacol.}

Le gaïacol est le plus sûr facteur pour régler la sensibilité de la teinture de gaïac.

Ceci ressort du tableau 4. Nous avons adopté la dose de 3 grammes de gaïacol. Cette teinture de gaïac nous permet de distinguer avec la plus grande certitude un lait qui a été chauffé à $78^{\circ}$. Une addition de $1 \%$ de lait cru est facilement décelée en se donnant comme règle d'écarter tout lait ayant réagi dans les 5 minutes. Normalement, le lait pasteurisé à $78^{\circ}$ donne une réaction positive en 10 à 20 minutes. Cette variation dépend principalement de l'exactitude de la lecture de la température de pasteurisation. Si la pasteurisation s'effectue à $77^{\circ} 3 / 4$ au lieu de $78^{\circ}$ la réaction devient positive en 7-8 minutes au lieu de 15 à 20 minutes.

\section{Rôle de la peroxydase.}

Nous savons que la peroxydase décompose l'eau oxygénée et que l'oxygène naissant formé oxyde certains éléments se trouvant dans la teinture de gaïac, en produisant des corps colorés. Il est très important de connaître les conditions exactes de la destruction de la peroxydase par la chaleur. Inıкноv [14], résumant les travaux de 
les données de la $3^{\mathrm{e}}$ colonne du tableau 4 . On constate que le lait chauffé à $79^{\circ}$ et le lait chauffé à $85^{\circ}$ pendant 5 minutes donnent tous les deux des réactions négatives, mais une addition dans ces laits de $5 \%$ de lait cru, provoque des réactions positives respectivement en 35 secondes et 10 minutes. Cette expérience prouve que le lait pasteurisé à $79^{\circ}$, tout en donnant une réaction négative, contient encore une certaine quantité de peroxydase.

De tels laits réagissent positivement avec la teinture de gaïac, quand ils contiennent $1 \%$ de lait cru.

Pour les mêmes raisons, les laits chauffés avee notre pasteurisateur à $78^{\circ}, 79^{\circ}$ et $80^{\circ}$, donnent une réaction négative et additionnés de $1 \%$ de lait eru ils donnent une réaction positive. D'autre part, comme nous l'avons montré, ces températures de chauffage sont suffisantes pour une bonne pasteurisation du lait.

Dans ces conditions, le contrôle biochimique (teinture de gaïac) pratiqué journellement sur tous les laits pasteurisés, complété par des analyses bactériologiques périodiques (tous les $2-3$ jours) garantit la production d'un vrai lait hygiénique.

\section{BIBLIOGRAPHIE.}

[1] A. Tapernoux. C. R. Soc. de Biol., CXIV, no 32, 1933.

[2] Pien et Baisse. Le Lait, no 139, 1934.

[3] J. Philippe. Thèse Doct. Vét., Lyon, 1935.

[4] M. Guituonneau. L'Industrie laitière, no 17, 1936.

[5] Kay et Graham. The Journal of Dairy Research, vol. VI, no 12, 1935.

[6] Orla-Jensen et Winter. Le Lait, no 143, 1935.

[7] G. Gorini. Le Lait, $\mathrm{n}^{0}$ 139, 1934.

[8] G. Gorinr. C. R. du $8^{\mathrm{e}}$ Congrès mondial de Laiterie à Rome, 1934.

[9] Chr. Barthes. Die Methoden zur Untersuchung von Milch und Molkereiprodukten. P. Parey, éditeur.

[10] Calmette, NÈgre et Boquet. Manuel technique de mierobiologie et sérologie. Masson \& Cie, édit.

[11] Clerc et Leibovitch. Congrès national d'Industrie laitière, Nancy, 1936.

[12] B. VAN DER BURG. Le Lait, no 117.

[13] MASEK. Le Lait, no, 159, 1936.

[14] G. S. Inıkнov. Biochirrie du lait (en russe), Léningrad.

\section{CONSIDÉRATIONS SUR LE LAIT CAILLÉ BULGARE DE BREBIS (1)}

\section{(Kisselo Mleko ou Kvasseno Mleko)}

par le Dr I. KVATCHKOFF

Nous nous proposons d'étudier dans ce travail le véritable lait aigre (kvasseno mleko) qu'on prépare exclusivement avec du lait de brebis. Le mot "yougourth", dont on se sert dans la littérature

(1) Revue d'Hygiène et de Médecine préventive, t. LVIII, $\mathrm{n}^{\circ} 6$, juin 1936. 